\title{
Analysis of Strong-Motion Signals from a M5.8 Earthquake

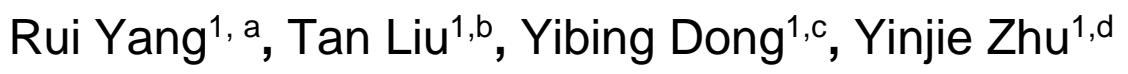 \\ ${ }^{1}$ Seismological Bureau of Hebei Province, China \\ a178515094@qq.com, b280613824@qq.com, cyuehun06@163.com, d526252372@qq.com
}

Keywords: strong-motion signal; PGA; acceleration response spectrum; seismic intensity Abstract. An earthquake of M5.8 occurred in Alxa of Inner Mongolia on April 15, 2015. There are 39 groups of strong-motion signals recorded by the National Strong Motion Network Center. PGA and instrumental intensity distribution characteristics is obtained from acceleration response spectrum analysis to the strong motion records. It can be concluded that the calculated instrumental intensity is basically consistent with the on-site disaster assessment, and it will provide rapid decision-making references for emergency rescue and disaster assessment.

\section{Introduction}

An M5.8 earthquake occurred in Alxa of Inner Mongolia $\left(39.8^{\circ} \mathrm{N}, 106.3^{\circ}\right.$ E) at 15:39 on April 15, 2015 with focal depth of $10 \mathrm{~km}$. The epicenter is near Bayan Muren, $43 \mathrm{~km}$ away from WuHai City, $86 \mathrm{~km}$ from Shizuishan City, and $121 \mathrm{~km}$ from Alxa Zuoqi, quite sparsely populated . To 08:00 April 17, seismic network of Inner Mongolia recorded aftershocks 86 times, M>3.0 accounts 3 times, and the greatest one is M4 .0 occurred at 15:44 on April 15. After the earthquake, the strong motion network center of China collected 13 records from the Seismological Bureau of Inner Mongolia, and 26s from Ningxia as well.

\section{Strong-motion observing system}

After the M5.8 earthquake, to 18:00, the strong motion network center had received 11 records from the Inner Mongolia network, and the number increased by 28 till 20:00, 2 of which from Inner Mongolia and 26s from NingXia. The triggered stations are shown in Table 1. The distribution of epicenter and stations is shown in Figure 1.

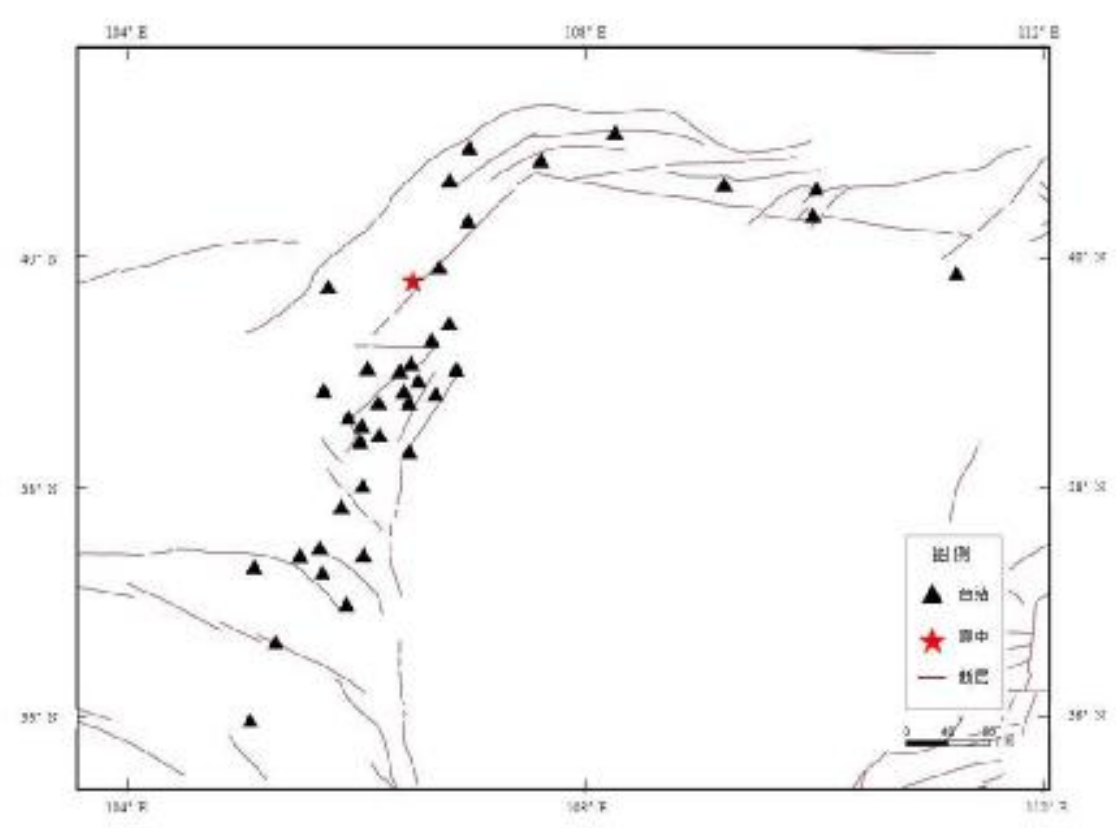

Figure 1. Distribution of the Epicenter and Stations 


\section{Data}

Figure 2 shows records from 2 stations nearest the epicenter, 15BYM and 15JLT. It can be seen that phases are clear to recognize, and after the M5.8, there' s another smaller earthquake recorded clearly by 15BYM. In 15JLT, its signal was mixed with the end waves of the former one.

(a)

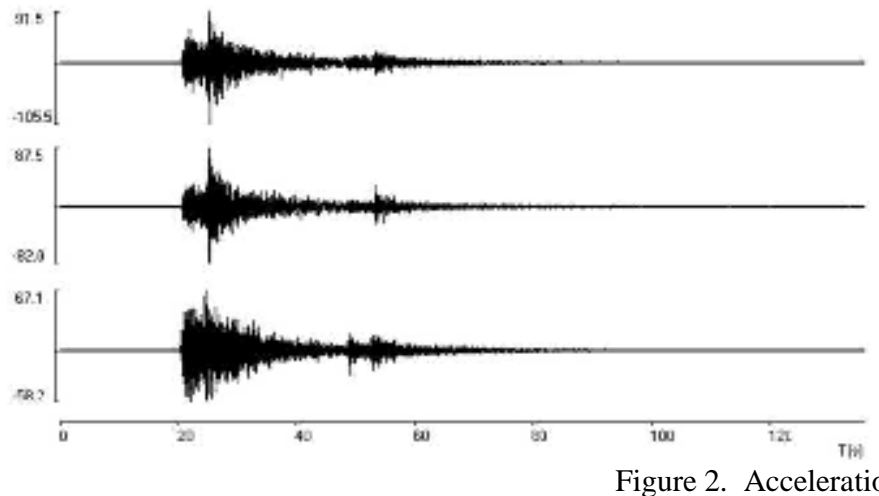

(b)

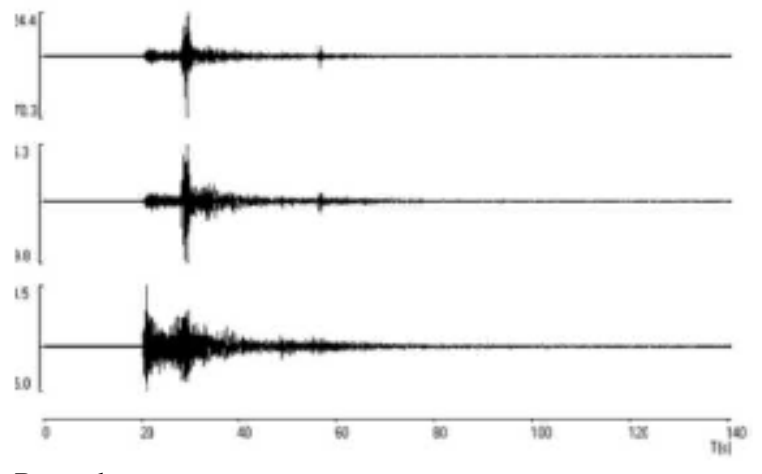

(a)15BYM; (b)15JLT

\section{Analysis}

Acceleration Response Spectrum. Acceleration response spectrum reflects characteristics of the seismic forces exerted on the structure from foundation[1]. Spectrum of the strong motion record shows the relationship between amplitude and frequency of vibration. Strong motion record contains rich frequencies, and the main frequency differs from region to region. It is necessary to analyze the frequency components of earthquake to protect constructions on sites[2]. Signals from 8 stations and 3 channels were selected to conduct SDOF acceleration spectral analysis. Figure 3 shows the results, where curves in different colors stand for spectrum with different damping ratio of $0,0.02,0.05,0.1$ and 0.2. It can be seen that all the records contain rich high-frequency components, and the dominant frequency of most horizontal channels distributes in $6-12 \mathrm{~Hz}$. The figure also shows the impact of different epicentral distance on the spectrum, the further from the epicenter, the more similar of the channels' spectrum. Besides, the attenuation of high-frequency components is more faster with distance increases.

Ground Motion Attenuation. Figure 4 shows the relationship between PGA and epicentral distance. It can be seen that PGA attenuation is not obvious within $100 \mathrm{~km}$, and after $100 \mathrm{~km}$, PGA of UD channel attenuate rapidly, near 400km, PGA of all channels attenuate to 0 quite rapidly.

Seismic intensity. After the M5.8 earthquake, Seismological Bureau of Inner Mongolia launched Earthquake Emergency Response III, and sent field teams to 72 points of 6 districts to conduct on-site disaster survey. The survey result is shown in Figure 5(a), the maximum intensity degree counts VII, the long axis strikes NEE, area above VI degree overs 5000km2. Figure 5(b) shows the distribution of Instrumental Intensity, with the same strike as figure(a). The main difference between (a) and (b) is the location of VII area, which depends on many factors including geology, instrumentation and site response etc[3]. Although not that accurate, the instrumental intensity can be calculated rapidly and automatically after a devastating earthquake, it will provide rapid decision-making references for emergency rescue and disaster assessment.

\section{Conclusions}

Strong motion records of M5.8 earthquake in Inner Mongolia were collected and analyzed. PGA and instrumental intensity distribution characteristics was obtained from acceleration response spectrum analysis to the strong motion records. It can be concluded that the calculated instrumental intensity is basically consistent with the on-site disaster assessment, and 
it will provide rapid decision-making references for emergency rescue and disaster assessment And the similarity can fully reflect the role and effect of the strong motion network.

(a)

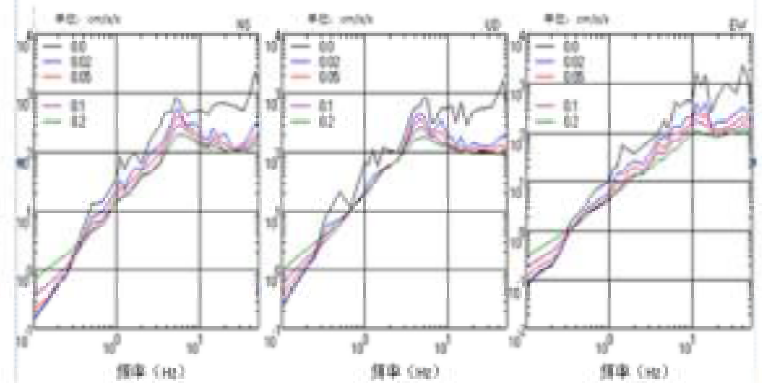

(c)

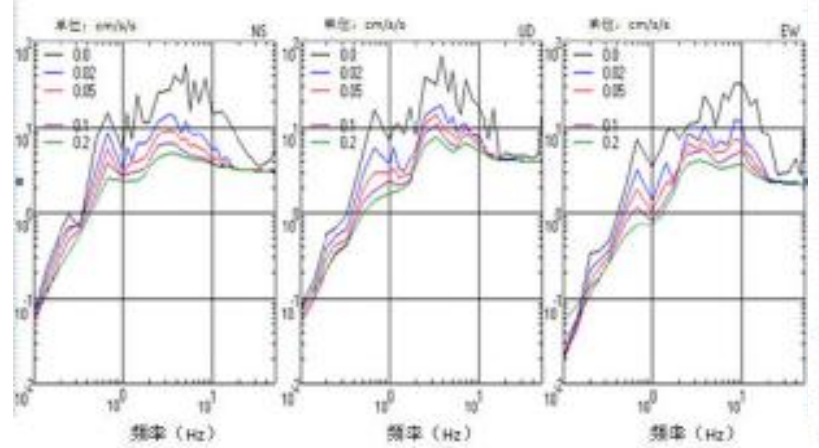

(e) (b)

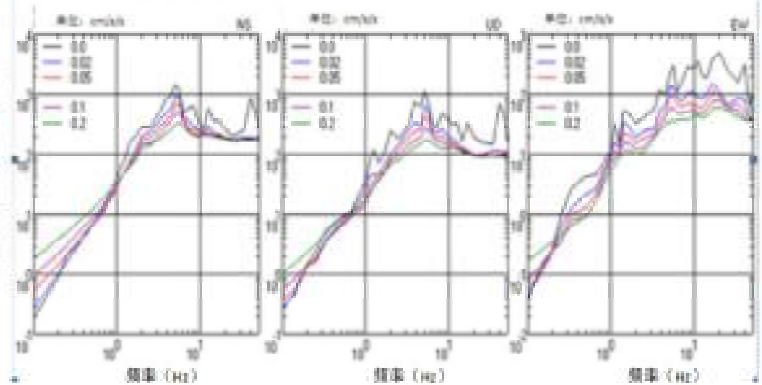

(d)

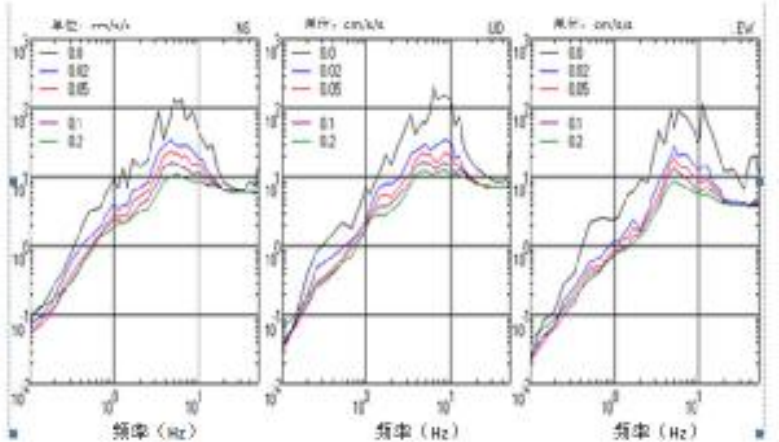

(f)

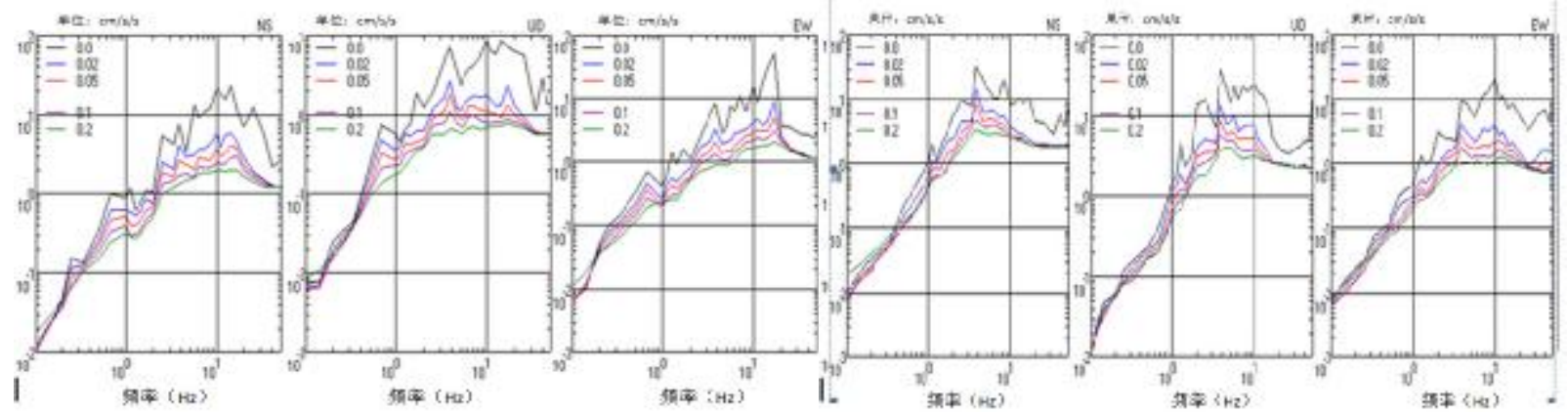

Figure 3. Acceleration Response Spectrum

(a) $15 \mathrm{BYM}$; (b) $15 \mathrm{JLT}$; (c) $15 \mathrm{BYT}$; (d) $15 \mathrm{WYT}$; (e) $15 \mathrm{BTT}$; (f) $15 \mathrm{XJW}$

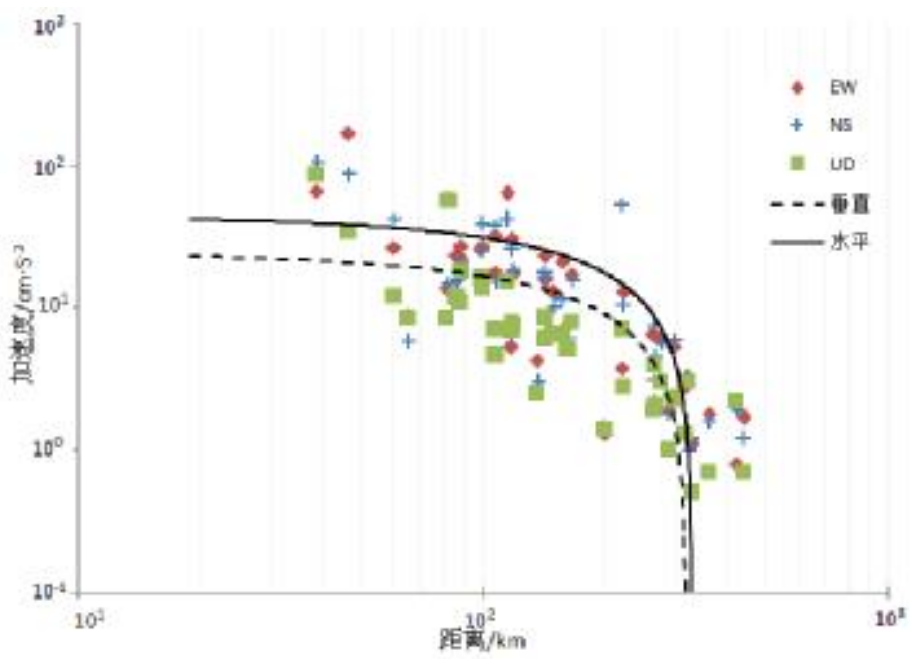

Figure 4. PGA Distribution 
(a)

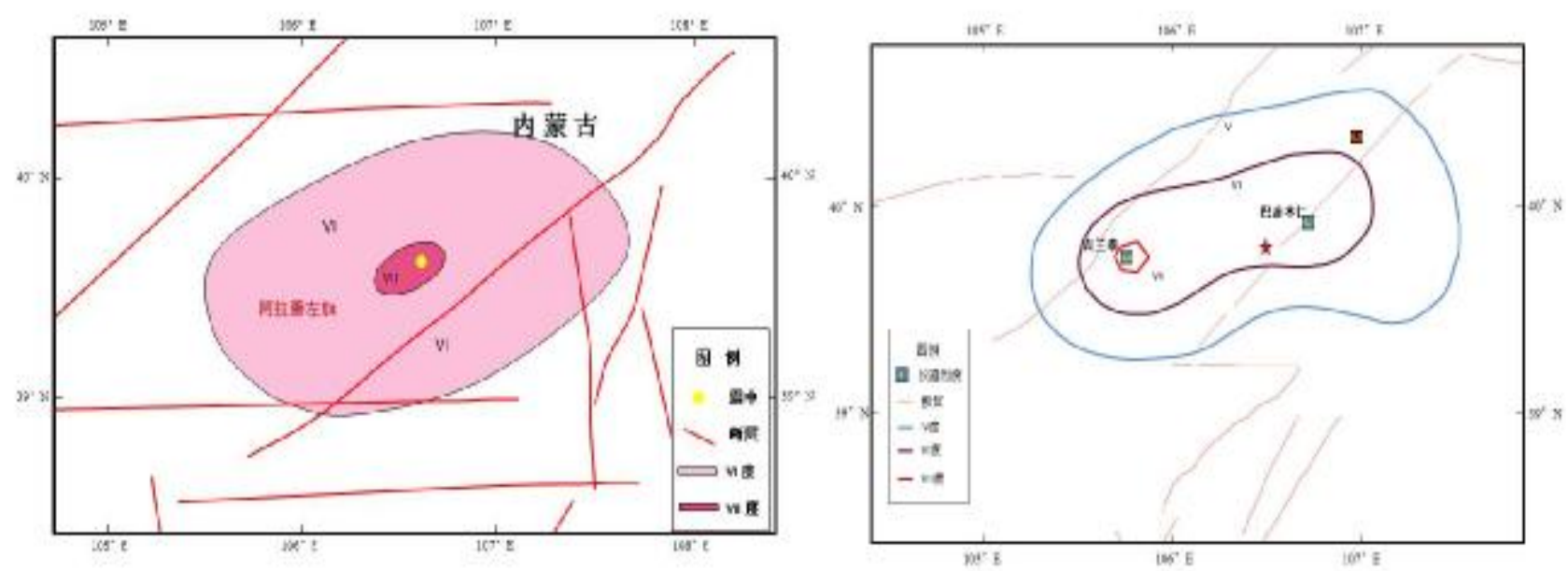

Figure 5. Intensity Distribution

(a)On-site Survey Intensity; (b) Instrumental Intensity

\section{Acknowledgments}

This work was financially supported by the Program of Seismological Bureau of Hebei Province (DZ20160407040).

\section{References}

[1] Wu Yih-Min,Shin Tzay-Chyn and Chang Chien-Hsin.Near Real-Time Mapping of Peak Ground Acceleration and Peak Ground Velocity Following a Strong Earthquake. Bulletin of the Seismological Society of America . 2001.

[2]J.W .David,V .Quitoriano,T .H .Heaton,H .Kanamori,C .W .Scrivner,C .B .Worden.TriNet"Shake Maps":RapidGenerationofPeakGroundMotionandIntensityMapsforEarthquakeinSouthernCalifo rnia. Earthquake Spectra . 1999.

[3] KanamoriH,P Maechling,E .Hauksson.ContinuousMonitoringofGround-MotionParameters. Bulletin of the Seismological Society of America . 1999. 